\title{
Erratum: Universal carrier thermoelectric-transport model based on percolation theory in organic semiconductors [Phys. Rev. B 91, 195205 (2015)]
}

Nianduan Lu, Ling Li, and Ming Liu

(Received 23 February 2017; published 17 March 2017)

DOI: 10.1103/PhysRevB.95.119904

In the original paper, $R^{3}$ was omitted in Eq. (8). Therefore, the parameter $R^{3}$ should be added in Eq. (8) as follows:

$$
\begin{aligned}
& P\left(Z_{m} \mid E_{i}\right) \\
& \quad=\frac{4 \pi R^{3}}{3(2 \alpha)^{3}}\left\{\begin{array}{l}
\int_{\varepsilon_{f}}^{\varepsilon_{i}}\left(S_{c}-\varepsilon_{j}+\varepsilon_{f}\right)^{3} g\left(\varepsilon_{j}\right) d \varepsilon_{j}+\int_{\varepsilon_{i}}^{S_{c}+\varepsilon_{f}}\left(S_{c}-\varepsilon_{j}+\varepsilon_{f}\right)^{3} g\left(\varepsilon_{j}\right) d \varepsilon_{j}+\int_{\varepsilon_{i}-S_{c}}^{\varepsilon_{f}}\left(S_{c}-\varepsilon_{i}+\varepsilon_{j}\right)^{3} g\left(\varepsilon_{j}\right) d \varepsilon_{j}, \quad \varepsilon_{i}>\varepsilon_{f}, \\
\int_{\varepsilon_{i}}^{\varepsilon_{f}}\left(S_{c}+\varepsilon_{i}-\varepsilon_{f}\right)^{3} g\left(\varepsilon_{j}\right) d \varepsilon_{j}+\int_{\varepsilon_{f}-S_{c}}^{\varepsilon_{i}}\left(S_{c}+\varepsilon_{i}-\varepsilon_{f}\right)^{3} g\left(\varepsilon_{j}\right) d \varepsilon_{j}+\int_{\varepsilon_{f}}^{\varepsilon_{i}+S_{c}}\left(S_{c}-\varepsilon_{j}+\varepsilon_{i}\right)^{3} g\left(\varepsilon_{j}\right) d \varepsilon_{j}, \quad \varepsilon_{i}<\varepsilon_{f} .
\end{array}\right.
\end{aligned}
$$

